\title{
ON INCREASING OF RESOLUTION OF SATELLITE IMAGES VIA THEIR FUSION WITH IMAGERY AT HIGHER RESOLUTION
}

\author{
Peter I. Kogut* Olga P. Kupenko ${ }^{\dagger \ddagger}$ Mykola V. Uvarov $§$
}

Communicated by Prof. P. O. Kasyanov

\begin{abstract}
In this paper we propose a new statement of the spatial increasing resolution problem of MODIS-like multi-spectral images via their fusion with Lansat-like imagery at higher resolution. We give a precise definition of the solution to the indicated problem, postulate assumptions that we impose at the initial data, establish existence and uniqueness result, and derive the corresponding necessary optimality conditions. For illustration, we supply the proposed approach by results of numerical simulations with real-life satellite images.
\end{abstract}

Key words: Satellite data fusion, image interpolation, image processing, variational approach, objective functional with non-standard growth conditions..

2010 Mathematics Subject Classification: 49Q20, 49K10, 49J45, 26 B30.

\section{Introduction}

Following in some aspects the paper [5], we propose a new variational approach to the spatial increasing resolution of multi spectral MODIS-like images via their fusion with Lansat-like imagery at higher resolution. Our approach is based on the variational model in Sobolev-Orlicz space with a non-standard growth condition of the objective functional and on the assumption that, to a large extent, the image topology in the each spectral channel is contained in the topographic map of its spectral energy. We discuss the well foundedness of the above approach, the consistency of the corresponding variational problem, and show that this problem admits a unique solution. We also derive some optimality conditions and supply our approach by results of numerical simulations with the real satellite images.

\footnotetext{
${ }^{*}$ Department of Differential Equations, Oles Honchar Dnipro National University, 72, Gagarin av., Dnipro, 49010, Ukraine; EOS Data Analytics Ukraine, Gagarin av., 103a, Dnipro, Ukraine p.kogut@i .ua, peter.kogut@eosda.com

${ }^{\dagger}$ Department of System Analysis and Control, Dnipro University of Technology 'Dnipro Polytechnics', 19, Yavornitskii av., 49005 Dnipro

${ }^{\ddagger}$ Institute Applied System Analysis, National Academy of Sciences and Ministry of Education and Science of Ukraine, 37/35, Peremogy av., IASA, 03056 Kyiv, Ukraine, kupenko.olga@gmail.com

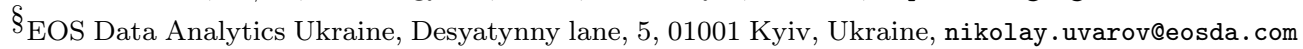

(C) P. I. Kogut, O. P. Kupenko, M. V. Uvarov, 2021.
} 


\section{Preliminaries}

We begin with some notation. Let $\Omega \subset \mathbb{R}^{2}$ be a bounded open set with a Lipschitz boundary $\partial \Omega$. Let $I: \Omega \rightarrow \mathbb{R}^{m}$, with $m \geqslant 3$, be a multispectral image containing the usual $R, G, B$ channels $I_{R}, I_{G}, I_{B}$, and arguably some others ones like the infrared channel $I_{N I R}$, i.e.,

$$
I(x)=\left[I_{R}(x), I_{G}(x), I_{B}(x), \ldots\right]^{t} \in \mathbb{R}^{m}, \quad \forall x \in \Omega .
$$

We say that $Y_{I}: \Omega \rightarrow \mathbb{R}$ is the panchromatic component of the multispectral image $I: \Omega \rightarrow \mathbb{R}^{m}$ (or in other words $Y_{I}$ is the spectral energy coming from the RGB-channels) if the following representation

$$
Y_{I}(x)=\alpha_{R} I_{R}(x)+\alpha_{G} I_{G}(x)+\alpha_{B} I_{B}(x), \quad \forall x \in \Omega
$$

holds for some weight coefficients $\alpha_{R}, \alpha_{G}, \alpha_{B} \geqslant 0$. In particular, if

$$
\alpha_{R}=0.299, \quad \alpha_{G}=0.587, \quad \alpha_{B}=0.114
$$

then $Y_{I}$ can be interpreted as the luma component of $I$ and it represents the perceptual brightness of the multispectral image $I: \Omega \rightarrow \mathbb{R}^{m}$.

For each $\lambda \in \mathbb{R}$, we define the upper level set of the spectral energy $Y_{I}$ as follows

$$
X_{\lambda}=\left\{x \in \Omega: Y_{I}(x) \geqslant \lambda\right\} .
$$

Then the spectral energy $Y_{I}$ can be recovered from its level sets by the reconstruction formula

$$
Y_{I}(x)=\sup \left\{\lambda: x \in X_{\lambda}\right\}, \quad \forall x \in \Omega .
$$

Hereinafter, we will refer to the family of connected components of the upper level sets of $Y_{I}$ as the topographic map of $Y_{I}$.

Let $S_{H} \subset \Omega$ and $S_{L} \subset \Omega$ be two sample grids on $\Omega$ such that

$$
\begin{aligned}
& S_{H}=\left\{\begin{array}{l|l}
\left(x_{i}, y_{j}\right) & \begin{array}{l}
x_{1}=x_{H}, x_{i}=x_{1}+\Delta_{H, x}(i-1), i=1, \ldots, N_{x}, \\
y_{1}=y_{H}, y_{j}=y_{1}+\Delta_{H, y}(j-1), j=1, \ldots, N_{y},
\end{array}
\end{array}\right\}, \\
& S_{L}=\left\{\begin{array}{l|l}
\left(x_{i}, y_{j}\right) & \begin{array}{l}
x_{1}=x_{L}, x_{i}=x_{1}+\Delta_{L, x}(i-1), i=1, \ldots, M_{x}, \\
y_{1}=y_{L}, y_{j}=y_{1}+\Delta_{L, y}(j-1), j=1, \ldots, M_{y},
\end{array}
\end{array}\right\},
\end{aligned}
$$

where $N_{x}>>M_{x}$ and $N_{y}>>M_{y}$.

Let $H: \Omega \rightarrow \mathbb{R}^{3}$ be a given multispectral (Landsat-like) image which is sampled at the grid of high resolution $S_{H}$. We suppose that, in practice, this image can be identified with an 3-D array

$$
H=\left\{\left[\begin{array}{l}
H_{R}\left(x_{i}, y_{j}\right) \\
H_{G}\left(x_{i}, y_{j}\right) \\
H_{B}\left(x_{i}, y_{j}\right)
\end{array}\right], i=1, \ldots, N_{x}, j=1, \ldots, N_{y}\right\} .
$$


Let $L: \Omega \rightarrow \mathbb{R}^{4}$ be a given multispectral (MODIS-like) image which is sampled at the grid of low resolution $S_{L}$, and it has 4 spectral channels $R, G, B$, and NIR. So, we can indentify this image with an 4 -D array

$$
L=\left\{\left[\begin{array}{c}
L_{R}\left(x_{i}, y_{j}\right) \\
L_{G}\left(x_{i}, y_{j}\right) \\
L_{B}\left(x_{i}, y_{j}\right) \\
L_{N I R}\left(x_{i}, y_{j}\right)
\end{array}\right], i=1, \ldots, M_{x}, j=1, \ldots, M_{y}\right\} .
$$

The problem, we are going to consider, can be formally stated as follows: Using only the data $H: S_{H} \rightarrow \mathbb{R}^{3}$ and $L: S_{L} \rightarrow \mathbb{R}^{4}$, we have to increase the resolution of the four-band image $L: S_{L} \rightarrow \mathbb{R}^{4}$ via its fusion with the three-band image $H: S_{H} \rightarrow \mathbb{R}^{3}$ at higher resolution such that the following properties for the retrieved high resolution multispectral image $I: S_{H} \rightarrow \mathbb{R}^{4}$ would be satisfied:

(i) The image $I: \Omega \rightarrow \mathbb{R}^{4}$ we are going to retrieve, should be of bounded variation, $I \in B V\left(\Omega ; \mathbb{R}^{4}\right)$.

(ii) The topographic maps for each spectral channel at higher resolution must have a similar structure to the topographic map of the spectral energy $Y_{H}$ coming from the RGB-channels of $H: S_{H} \rightarrow \mathbb{R}^{3}$.

(iii) The spectral energies $Y_{I}$ and $Y_{H}$ should be as close as possible with respect to the $L^{2}(\Omega)$-norm.

(iv) The sampled values of $I: \Omega \rightarrow \mathbb{R}^{4}$ on the grid of low resolution $S_{L}$ should be as close as possible in $L^{2}$-metric to the multispectral imagery $L: S_{L} \rightarrow \mathbb{R}^{4}$.

(v) The NIR-channel $I_{N I R}$ for the retrieved high resolution multispectral image $I: \Omega \rightarrow \mathbb{R}^{4}$ should be in the same regression relationship with $I_{R}, I_{G}, I_{B}$ channels as $L_{N I R}$ with $L_{R}, L_{G}, L_{B}$, that is, if

$L_{N I R}\left(x_{i}, y_{j}\right)=\gamma_{R} L_{R}\left(x_{i}, y_{j}\right)+\gamma_{G} L_{G}\left(x_{i}, y_{j}\right)+\gamma_{B} L_{B}\left(x_{i}, y_{j}\right), \quad \forall\left(x_{i}, y_{j}\right) \in S_{L}$

is a linear regression model which is fitted using the least squares approach, then

$I_{N I R}\left(x_{i}, y_{j}\right)=\gamma_{R} I_{R}\left(x_{i}, y_{j}\right)+\gamma_{G} I_{G}\left(x_{i}, y_{j}\right)+\gamma_{B} I_{B}\left(x_{i}, y_{j}\right), \quad \forall\left(x_{i}, y_{j}\right) \in S_{H}$

with the same regression coefficients $\gamma_{R}, \gamma_{G}, \gamma_{B} \in \mathbb{R}$. 


\section{Auxiliaries}

\section{1. $B V$-Space}

By $B V(\Omega)$ we denote the space of all functions $u \in L^{1}(\Omega)$ for which their distributional derivatives are representable by finite Borel measures in $\Omega$, i.e.

$$
\int_{\Omega} u \frac{\partial \phi}{\partial x_{i}} d x=-\int_{\Omega} \phi d D_{i} u, \quad \forall \phi \in C_{0}^{\infty}(\Omega), i=1,2
$$

for some $\mathbb{R}^{2}$-valued measure $D u=\left(D_{1} u, D_{2} u\right) \in \mathcal{M}^{2}(\Omega)$. It can be shown that $B V(\Omega)$, endowed with the norm

$$
\|u\|_{B V(\Omega)}=\|u\|_{L^{1}(\Omega)}+|D u|(\Omega)
$$

is a Banach space, where

$$
\begin{aligned}
|D u|(\Omega): & =\int_{\Omega} d|D u| \\
& =\sup \left\{\int_{\Omega} u \operatorname{div} \varphi d x: \varphi \in C_{0}^{1}\left(\Omega ; \mathbb{R}^{2}\right),|\varphi(x)| \leqslant 1 \text { for } x \in \Omega\right\}
\end{aligned}
$$

stands for the total variation of $u$ in $\Omega$. It is clear that $|D u|(\Omega)=\int_{\Omega}|\nabla u| d x$ if $u$ is continuously differentiable in $\Omega$.

The following embedding results for $B V$-function plays a crucial role for qualitative analysis of variational problems that we study in this paper.

Proposition 3.1. $\left[4\right.$, p.378] Let $\Omega$ be an open bounded Lipschitz subset of $\mathbb{R}^{2}$. Then the embedding $B V\left(\Omega ; \mathbb{R}^{M}\right) \hookrightarrow L^{2}\left(\Omega ; \mathbb{R}^{M}\right)$ is continuous and the embeddings $B V\left(\Omega ; \mathbb{R}^{M}\right) \hookrightarrow L^{p}\left(\Omega ; \mathbb{R}^{M}\right)$ are compact for all $p$ such that $1 \leqslant p<2$. Moreover, there exists a constant $C_{e m}>0$ which depends only on $\Omega$ and $p$ such that for all $u$ in $B V\left(\Omega ; \mathbb{R}^{M}\right)$,

$$
\left(\int_{\Omega}|u|^{p} d x\right)^{1 / p} \leqslant C_{e m}\|u\|_{B V\left(\Omega ; \mathbb{R}^{M}\right)}, \quad \forall p \in[1,2] .
$$

According to the Radon-Nikodym theorem, if $u \in B V(\Omega)$ then there exists $\nabla u \in L^{1}\left(\Omega ; \mathbb{R}^{2}\right)$ and a measure $D_{s} u$ singular with respect to the 2-dimensional Lebesgue measure $\mathcal{L}^{2}\left\llcorner\Omega\right.$ restricted to $\Omega$, such that $D u=\nabla u \mathcal{L}^{2}\left\llcorner\Omega+D_{s} u\right.$.

We recall that if $u \in B V(\Omega)$, then almost all its level sets $\{x \in \Omega: u(x) \geqslant \lambda\}$ are sets of finite perimeter. Hence, at almost all points of almost all level sets of $u \in B V(\Omega)$ we may define a normal vector $\theta(x)$. This vector field of normals $\theta(x)$ can be also defined as the Radon-Nikodym derivative of the measure $D u$ with respect to $|D u|$, i.e., it formally satisfies the following relations

$$
(\theta, D u)=|D u| \text { and }|\theta| \leqslant 1 \text { a.e. in } \Omega \text {. }
$$

In the sequel, we will refer to the vector field $\theta$ as the vector field of unit normals to the topographic map of a function $u$. Further information on $B V$-functions and their properties can be found in $[1,4]$. 
Remark 3.1. In practice, at the discrete level, $\theta(x, y)$ can be defined by the rule $\theta\left(x_{i}, y_{j}\right)=\frac{D u\left(x_{i}, y_{j}\right)}{\left|D u\left(x_{i}, y_{j}\right)\right|}$ when $D u\left(x_{i}, y_{j}\right) \neq 0$, and $\theta=0$ when $D u\left(x_{i}, y_{j}\right)=0$. However, as was mentioned in [5], a better choice for $\theta(x, y)$ would be to compute it as $\xi(t)=\frac{D U(t, \cdot)}{D U(t, \cdot)}$ for some small value of $t>0$, where $U(t, x, y)$ is a solution of the following initial-boundary value problem with $1 D$-Laplace operator in the right hand side

$$
\begin{aligned}
& \frac{\partial U}{\partial t}=\operatorname{div}\left(\frac{D U}{|D U|}\right), \quad t \in(0,+\infty),(x, y) \in \Omega, \\
& U(0, x, y)=u(x, y), \quad(x, y) \in \Omega, \\
& \frac{\partial U(0, x, y)}{\partial \nu}=0, \quad t \in(0,+\infty), \quad(x, y) \in \partial \Omega .
\end{aligned}
$$

As a result, for any $t>0$, there can be found a vector field

$$
\xi \in L^{\infty}\left(\Omega ; \mathbb{R}^{2}\right) \text { with }\|\xi(t)\|_{L^{\infty}\left(\Omega ; \mathbb{R}^{2}\right)} \leqslant 1
$$

such that

$$
(\xi(t), U(t, \cdot))=|D U(t, \cdot)| \text { in } \Omega, \quad \xi(t) \cdot \nu=0 \text { on } \partial \Omega,
$$

and $U_{t}(t, x, y)=\operatorname{div} \xi(t, x, y)$ in the sense of distributions on $\Omega$ for a.a. $t>0$.

We notice that following this procedure, for small value of $t>0$, we do not distort the geometry of the function $u(x, y)$ in an essential way. Moreover, it can be shown that this regularization of the vector field $\theta(x, y)=\frac{D U(x, y)}{|D U(x, y)|}$ satisfies condition $\operatorname{div} \theta \in L^{2}(\Omega)$.

\subsection{On Orlicz Spaces}

Let $p(\cdot)$ be a measurable exponent function on $\Omega$ such that

$$
1 \leqslant \alpha \leqslant p(x) \leqslant \beta<\infty \quad \text { a.e. in } \Omega,
$$

where $\alpha$ and $\beta$ are given constants. Let $p^{\prime}(\cdot)=\frac{p(\cdot)}{p(\cdot)-1}$ be the corresponding conjugate exponent. It is clear that

$$
1 \leqslant \underbrace{\frac{\beta}{\beta-1}}_{\beta^{\prime}} \leqslant p^{\prime}(x) \leqslant \underbrace{\frac{\alpha}{\alpha-1}}_{\alpha^{\prime}} \text { a.e. in } \Omega,
$$

where $\beta^{\prime}$ and $\alpha^{\prime}$ stand for the conjugates of constant exponents. Denote by $L^{p(\cdot)}(\Omega)$ the set of all measurable functions $f(x)$ on $\Omega$ such that $\int_{\Omega}|f(x)|^{p(x)} d x<\infty$. Then $L^{p(\cdot)}(\Omega)$ is a reflexive separable Banach space with respect to the Luxemburg norm (see $[7,8]$ for the details)

$$
\|f\|_{L^{p(\cdot)}(\Omega)}=\inf \left\{\lambda>0: \rho_{p}\left(\lambda^{-1} f\right) \leqslant 1\right\},
$$


where $\rho_{p}(f):=\int_{\Omega}|f(x)|^{p(x)} d x$.

It is well-known that $L^{p(\cdot)}(\Omega)$ is reflexive provided $\alpha>1$, and its dual is $L^{p^{\prime}(\cdot)}(\Omega)$, that is, any continuous functional $F=F(f)$ on $L^{p(\cdot)}(\Omega)$ has the form (see [21, Lemma 13.2])

$$
F(f)=\int_{\Omega} f g d x, \quad \text { with } g \in L^{p^{\prime}(\cdot)}(\Omega) .
$$

As for the infimum in (3.8), we have the following result.

Proposition 3.2. The infimum in (3.8) is attained if $\rho_{p}(f)>0$. Moreover,

$$
\text { if } \lambda_{*}:=\|f\|_{L^{p(\cdot)(\Omega)}}>0, \text { then } \rho_{p}\left(\lambda_{*}^{-1} f\right)=1 .
$$

Taking this result and condition $1 \leqslant \alpha \leqslant p(x) \leqslant \beta$ into account, we see that

$$
\begin{gathered}
\frac{1}{\lambda_{*}^{\beta}} \int_{\Omega}|f(x)|^{p(x)} d x \leqslant \int_{\Omega}\left|\frac{f(x)}{\lambda_{*}}\right|^{p(x)} d x \leqslant \frac{1}{\lambda_{*}^{\alpha}} \int_{\Omega}|f(x)|^{p(x)} d x, \\
\frac{1}{\lambda_{*}^{\beta}} \int_{\Omega}|f(x)|^{p(x)} d x \leqslant 1 \leqslant \frac{1}{\lambda_{*}^{\alpha}} \int_{\Omega}|f(x)|^{p(x)} d x .
\end{gathered}
$$

provided $\lambda_{*} \geqslant 1$. And we arrive at the reverse inequality if $0<\lambda_{*}<1$. Hence, (see $[7,8,20]$ for the details)

$$
\begin{aligned}
& \|f\|_{L^{p(\cdot)}(\Omega)}^{\alpha} \leqslant \int_{\Omega}|f(x)|^{p(x)} d x \leqslant\|f\|_{L^{p(\cdot)}(\Omega)}^{\beta}, \text { if }\|f\|_{L^{p(\cdot)}(\Omega)}>1, \\
& \|f\|_{L^{p(\cdot)}(\Omega)}^{\beta} \leqslant \int_{\Omega}|f(x)|^{p(x)} d x \leqslant\|f\|_{L^{p(\cdot)}(\Omega)}^{\alpha}, \text { if }\|f\|_{L^{p(\cdot)}(\Omega)}<1,
\end{aligned}
$$

and, therefore,

$$
\begin{gathered}
\|f\|_{L^{p(\cdot)}(\Omega)}^{\alpha}-1 \leqslant \int_{\Omega}|f(x)|^{p(x)} d x \leqslant\|f\|_{L^{p(\cdot)}(\Omega)}^{\beta}+1, \quad \forall f \in L^{p(\cdot)}(\Omega), \\
\|f\|_{L^{p(\cdot)}(\Omega)}=\int_{\Omega}|f(x)|^{p(x)} d x, \text { if }\|f\|_{L^{p(\cdot)}(\Omega)}=1 .
\end{gathered}
$$

The following estimates are well-known (see, for instance, $[7,8,20]$ ): if $f \in$ $L^{p(\cdot)}(\Omega)$ then

$$
\begin{gathered}
\|f\|_{L^{\alpha}(\Omega)} \leqslant(1+|\Omega|)^{1 / \alpha}\|f\|_{L^{p(\cdot)}(\Omega)}, \\
\|f\|_{L^{p(\cdot)(\Omega)}} \leqslant(1+|\Omega|)^{1 / \beta^{\prime}}\|f\|_{L^{\beta}(\Omega)}, \quad \beta^{\prime}=\frac{\beta}{\beta-1}, \quad \forall f \in L^{\beta}(\Omega) .
\end{gathered}
$$

Let $\left\{f_{k} \in L^{p(\cdot)}(\Omega)\right\}_{k \in \mathbb{N}}$ be a given sequence, where the exponent $p \in C(\bar{\Omega})$ satisfies property (3.6). We say that the sequence $\left\{f_{k} \in L^{p(\cdot)}(\Omega)\right\}_{k \in \mathbb{N}}$ is bounded if (see [15, Section 6.2])

$$
\limsup _{k \rightarrow \infty} \int_{\Omega}\left|f_{k}(x)\right|^{p(x)} d x<+\infty .
$$


Definition 3.1. A bounded sequence $\left\{f_{k} \in L^{p(\cdot)}(\Omega)\right\}_{k \in \mathbb{N}}$ is weakly convergent in the Orlicz space $L^{p(\cdot)}(\Omega)$ to a function $f \in L^{p(\cdot)}(\Omega)$, if

$$
\lim _{k \rightarrow \infty} \int_{\Omega} f_{k} \varphi d x=\int_{\Omega} f \varphi d x, \quad \forall \varphi \in C_{0}^{\infty}\left(\mathbb{R}^{2}\right) .
$$

For our further analysis, we make use of the following lower semicontinuity property of the $L^{p(\cdot)}$-norm with respect to the weak convergence in $L^{p(\cdot)}(\Omega)$ (we refer to [21, Lemma 13.3] for details).

Proposition 3.3. If a bounded sequence $\left\{f_{k} \in L^{p(\cdot)}(\Omega)\right\}_{k \in \mathbb{N}}$ converges weakly in $L^{\alpha}(\Omega)$ to $f$, where $\alpha>1$ is defined in (3.6), then $f \in L^{p(\cdot)}(\Omega), f_{k} \rightarrow f$ in $L^{p(\cdot)}(\Omega)$, and

$$
\liminf _{k \rightarrow \infty} \int_{\Omega}\left|f_{k}(x)\right|^{p(x)} d x \geqslant \int_{\Omega}|f(x)|^{p(x)} d x
$$

Remark 3.2. Arguing in a similar manner as in [21, Lemma 13.3] and using the estimate

$$
\liminf _{k \rightarrow \infty} \int_{\Omega} \frac{1}{p_{k}(x)}\left|f_{k}(x)\right|^{p_{k}(x)} d x \geqslant \int_{\Omega} f(x) \varphi(x) d x-\int_{\Omega} \frac{1}{p_{k}^{\prime}(x)}|\varphi(x)|^{p^{\prime}(x)} d x,
$$

which is valid for any smooth function $\varphi$, it is easy to show that the lower semicontinuity property (3.17) can be generalized as follows

$$
\liminf _{k \rightarrow \infty} \int_{\Omega} \frac{1}{p(x)}\left|f_{k}(x)\right|^{p(x)} d x \geqslant \int_{\Omega} \frac{1}{p(x)}|f(x)|^{p(x)} d x .
$$

We need the following result that leads to the analog of the Hölder inequality in Lebesgue spaces with variable exponents (for the details we refer to $[7,8]$ ).

Proposition 3.4. If $f \in L^{p(\cdot)}(\Omega)^{N}$ and $g \in L^{p^{\prime}(\cdot)}(\Omega)^{N}$, then $(f, g) \in L^{1}(\Omega)$ and

$$
\int_{\Omega}(f, g) d x \leqslant 2\|f\|_{L^{p(\cdot)}(\Omega)^{N}}\|g\|_{L^{p^{\prime}(\cdot)}(\Omega)^{N}} .
$$

\subsection{Sobolev Spaces with Variable Exponent}

We recall here the well-known facts concerning the Sobolev spaces with variable exponent. Let $p(\cdot)$ be a measurable exponent function on $\Omega$ such that $1<\alpha \leqslant$ $p(x) \leqslant \beta<\infty$ a.e. in $\Omega$, where $\alpha$ and $\beta$ are given constants. We associate with it the so-called Sobolev-Orlicz space

$$
W^{1, p(\cdot)}(\Omega):=\left\{y \in W^{1,1}(\Omega): \int_{\Omega}\left[|y(x)|^{p(x)}+|\nabla y(x)|^{p(x)}\right] d x<+\infty\right\}
$$

and equip it with the norm $\|y\|_{W_{0}^{1, p(\cdot)}(\Omega)}=\|y\|_{L^{p(\cdot)}(\Omega)}+\|\nabla y\|_{L^{p(\cdot)}\left(\Omega ; \mathbb{R}^{2}\right)}$. 
It is well-known that, in general, unlike classical Sobolev spaces, smooth functions are not necessarily dense in $W=W_{0}^{1, p(\cdot)}(\Omega)$. Hence, with the given variable exponent $p=p(x)(1<\alpha \leqslant p \leqslant \beta)$ there can be associated another Sobolev space with variable exponent,

$$
H=H^{1, p(\cdot)}(\Omega) \text { as the closure of the set } C^{\infty}(\bar{\Omega}) \text { in } W^{1, p(\cdot)}(\Omega) \text {-norm. }
$$

Since the identity $W=H$ is not always valid, it makes sense to say that an exponent $p(x)$ is regular if $C^{\infty}(\bar{\Omega})$ is dense in $W^{1, p(\cdot)}(\Omega)$.

The following result reveals an important property ensuring the regularity of exponent $p(x)$.

Proposition B.1. Assume that there exists $\delta \in(0,1]$ such that $p \in C^{0, \delta}(\bar{\Omega})$. Then the set $C^{\infty}(\bar{\Omega})$ is dense in $W^{1, p(\cdot)}(\Omega)$, and, therefore, $W=H$.

Proof. Let $p \in C^{0, \delta}(\bar{\Omega})$ be a given exponent. Since

$$
\lim _{t \rightarrow 0}|t|^{\delta} \log (|t|)=0 \quad \text { with an arbitrary } \delta \in(0,1],
$$

it follows from the Hölder continuity of $p(\cdot)$ that

$$
|p(x)-p(y)| \leqslant C|x-y|^{\delta} \leqslant\left[\sup _{x, y \in \Omega}|| x-\left.y\right|^{\delta} \log \left(|x-y|^{-1}\right)\right] \omega(|x-y|), \forall x, y \in \Omega \text {, }
$$

where $\omega(t)=C / \log \left(|t|^{-1}\right)$, and $C>0$ is some positive constant.

Then property (3.21) implies that $p(\cdot)$ is a log-Hölder continuous function. So, to deduce the density of $C^{\infty}(\bar{\Omega})$ in $W^{1, p(\cdot)}(\Omega)$ it is enough to refer to Theorem 13.10 in [21].

\section{Main Assumptions}

Let $H: S_{H} \rightarrow \mathbb{R}^{3}$ and $L: S_{L} \rightarrow \mathbb{R}^{4}$ be given digital images. Hereinafter, we assume that their continuous counterparts $H: \Omega \rightarrow \mathbb{R}^{3}$ and $L: \Omega \rightarrow \mathbb{R}^{4}$ are such that

$$
Y_{H} \in B V(\Omega) \text { and } Y_{L} \in L^{2}(\Omega),
$$

where $Y_{H}$ and $Y_{L}$ stand for the spectral energies of the $H$ and $L$ images, respectively.

Before proceed further, we associate with the spectral energy $Y_{H}$ the so-called texturity characteristic $p: \Omega \rightarrow \mathbb{R}$ following the rule

$$
p(x):=\mathfrak{F}\left(Y_{H}(x)\right)=1+g\left(\left|\left(\nabla G_{\sigma} * Y_{H}\right)(x)\right|\right), \quad \forall x \in \Omega,
$$

where $g:[0, \infty) \rightarrow(0, \infty)$ is the edge-stopping function which we take it in the form of the Cauchy law $g(t)=\frac{1}{1+(t / a)^{2}}$ with an appropriate $a>0,\left(\nabla G_{\sigma} * Y_{H}\right)(x)$ determines the convolution of function $Y_{H}$ with the two-dimensional Gaussian filter kernel $G_{\sigma}$,

$$
\begin{gathered}
G_{\sigma}(x)=\frac{1}{2 \pi \sigma^{2}} e^{-\frac{|x|^{2}}{2 \sigma^{2}}}, \quad x \in \mathbb{R}^{2} \\
\left(\nabla G_{\sigma} * Y_{H}\right)(x):=\int_{\Omega} \nabla G_{\sigma}(x-y) Y_{H}(y) d y, \quad \forall x \in \Omega .
\end{gathered}
$$


Here, the parameter $\sigma>0$ determines the spatial size of the image details which are removed by this $2 D$ filter.

Since the magnitude $g\left(\left|\left(\nabla G_{\sigma} * Y_{H}\right)(x)\right|\right)$ is close to one at those points, where the spectral energy $Y_{H}$ is slowly varying, and this value is close to zero at the edges of $Y_{H}$, it follows that the function $p(x)$ can be interpreted as a texturity characteristic of the panchromatic image $Y_{H}$.

The following result plays a crucial role in the sequel.

Lemma 4.1. Let $Y_{H} \in L^{1}(\Omega)$ be a given spectral energy. Let

$$
p=1+g\left(\left|\left(\nabla G_{\sigma} * Y_{H}\right)\right|\right)
$$

be the corresponding texturity characteristic. Then

$$
\begin{gathered}
p \in C^{0,1}(\bar{\Omega}), \\
\alpha:=1+\delta \leqslant p(x) \leqslant \beta:=2, \quad \forall x \in \Omega,
\end{gathered}
$$

where $\delta=\frac{a^{2}}{a^{2}+\left\|G_{\sigma}\right\|_{C^{1}(\overline{\Omega-\Omega})}^{2}\left\|Y_{H}\right\|_{L^{1}(\Omega)}^{2}}$.

Proof. By smoothness of the Gaussian filter kernel $G_{\sigma}$, we have

$$
\begin{aligned}
\left|\left(\nabla G_{\sigma} * Y_{H}\right)(x)\right| & \leqslant \int_{\Omega}\left|\nabla G_{\sigma}(x-y)\right| Y_{H}(y) d y \leqslant\left\|G_{\sigma}\right\|_{C^{1}(\overline{\Omega-\Omega})}\left\|Y_{H}\right\|_{L^{1}(\Omega)}, \\
p(x) & =1+\frac{a^{2}}{a^{2}+\left(\left|\left(\nabla G_{\sigma} * Y_{H}\right)(x)\right|\right)^{2}} \\
& \geqslant 1+\frac{a^{2}}{a^{2}+\left\|G_{\sigma}\right\|_{C^{1}(\overline{\Omega-\Omega})}^{2}\left\|Y_{H}\right\|_{L^{1}(\Omega)}^{2}}, \quad \forall x \in \Omega .
\end{aligned}
$$

From this the existence of a positive value $\delta \in(0,1)$ such that estimate (4.6) holds true follows. Combining this fact with $\max _{x \in \bar{\Omega}}|p(x)| \leqslant \beta:=2$, we arrive at the announced estimate (4.6).

As for the property (4.5), we make use of the estimate

$$
\begin{array}{r}
|p(x)-p(y)| \leqslant a^{2}\left|\frac{\left|\left(\nabla G_{\sigma} * Y_{H}\right)(x)\right|^{2}-\left|\left(\nabla G_{\sigma} * Y_{H}\right)(y)\right|^{2}}{\left(a^{2}+\left|\left(\nabla G_{\sigma} * Y_{H}\right)(x)\right|^{2}\right)\left(a^{2}+\left|\left(\nabla G_{\sigma} * Y_{H}\right)(y)\right|^{2}\right)}\right| \\
\leqslant \frac{2\left\|G_{\sigma}\right\|_{C^{1}(\overline{\Omega-\Omega})}\left\|Y_{H}\right\|_{L^{1}(\Omega)}}{a^{2}}||\left(\nabla G_{\sigma} * Y_{H}\right)(x)|-|\left(\nabla G_{\sigma} * Y_{H}\right)(y)|| \\
\leqslant \frac{2\left\|G_{\sigma}\right\|_{C^{1}(\overline{\Omega-\Omega})} \gamma_{1}^{2}|\Omega|}{a^{2}} \int_{\Omega}\left|\nabla G_{\sigma}(x-z)-\nabla G_{\sigma}(y-z)\right| d z, \forall x, y \in \Omega,
\end{array}
$$

with $\gamma_{1}=\max _{x \in \Omega}\left|Y_{H}(x)\right|$. Then, by smoothness of the function $\nabla G_{\sigma}(\cdot)$, we deduce: there exists a positive constant $C_{G}>0$ such that

$$
|p(x)-p(y)| \leqslant \frac{2\left\|G_{\sigma}\right\|_{C^{1}(\overline{\Omega-\Omega})} \gamma_{1}^{2}|\Omega| C_{G}}{a^{2}}|x-y|, \forall x, y \in \Omega .
$$


Setting $C:=\frac{2\left\|G_{\sigma}\right\|_{C^{1}(\overline{\Omega-\Omega})} \gamma_{1}^{2}|\Omega| C_{G}}{a^{2}}$, we finally see that

$$
p(\cdot) \in \mathfrak{S}=\left\{h \in C^{0,1}(\Omega) \mid \begin{array}{c}
|h(x)-h(y)| \leqslant C|x-y|, \forall x, y, \in \Omega, \\
1<\alpha \leqslant h(\cdot) \leqslant \beta \text { in } \bar{\Omega} .
\end{array}\right\}
$$

The algorithm that we propose to realize for the spatial interpolation of MODIS-like multi-band color images to the Landsat-like imagery at high resolution, is essentially grounded on the following key assumptions.

Assumption 1. The MODIS image $L: S_{L} \rightarrow \mathbb{R}^{4}$ and the Landsat image $H$ : $S_{H} \rightarrow \mathbb{R}^{3}$ are rigidly co-registered.

This means that there exists an affine transformation $\mathcal{F}: \mathbb{R}^{2} \rightarrow \mathbb{R}^{2}$ of the form

$$
\mathcal{F}(x)=B x+a, \quad \forall x \in \mathbb{R}^{2},
$$

where

$$
a=\left[\begin{array}{l}
a_{1} \\
a_{2}
\end{array}\right] \quad \text { and } \quad=\left[\begin{array}{ll}
b_{11} & b_{12} \\
b_{21} & b_{22}
\end{array}\right]
$$

such that the MODIS-like image after the affine transformation $L\left(\mathcal{F}^{-1}(\cdot)\right): S_{L} \rightarrow$ $\mathbb{R}^{4}$ and Landsat-like image $H: S_{H} \rightarrow \mathbb{R}^{3}$, after the bilinear resampling to the grid of low resolution $S_{L}$, could be successfully matched.

In practice, the co-registration procedure can be realized using, for instance, the open-source LSReg v2.0.2 software $[16,19]$ that has been used in a number of recent studies $[9,17]$, or the rigid co-registration approach that was recently developed by the EOS Company [11,12]. However, in both cases, in order to find an appropriate affine transformation, we propose to apply the above mentioned procedure not to the original images, but rather to their spectral energies

$$
Y_{L}\left(x_{i}, y_{j}\right)=\alpha_{R} L_{R}\left(x_{i}, y_{j}\right)+\alpha_{G} L_{G}\left(x_{i}, y_{j}\right)+\alpha_{B} L_{B}\left(x_{i}, y_{j}\right), \quad \forall\left(x_{i}, y_{j}\right) \in S_{L}
$$

and

$$
Y_{H}\left(x_{i}, y_{j}\right)=\alpha_{R} H_{R}\left(x_{i}, y_{j}\right)+\alpha_{G} H_{G}\left(x_{i}, y_{j}\right)+\alpha_{B} H_{B}\left(x_{i}, y_{j}\right), \quad \forall\left(x_{i}, y_{j}\right) \in S_{H},
$$

where the last one should be previously resampled to the grid of low resolution $S_{L}$

Assumption 2. The low resolution pixels in the image $L: S_{L} \rightarrow \mathbb{R}^{4}$ are formed from the high resolution pixels of $I: S_{H} \rightarrow \mathbb{R}^{4}$ by a low pass filtering (the se-called subsampling procedure). 
As a consequence of this Assumption, we can suppose that there exists an impulse response $K$ such that

$$
L\left(x_{i}, y_{j}\right)=[\mathcal{K} * I]\left(x_{i}, y_{j}\right), \quad \forall i=1, \ldots, M_{x}, \forall j=1, \ldots, M_{y} .
$$

where $[\mathcal{K} * I]$ stands for the convolution operator. In particular, if $\mathcal{K}=\left[k_{p, q}\right]_{p, q=1, \ldots, K}$ is a squared matrix, then

$$
[\mathcal{K} * I]\left(x_{i}, y_{j}\right)=\sum_{p=1}^{K} \sum_{q=1}^{K} k_{p, q} I\left(x_{i-p+1}, y_{j-q+1}\right)
$$

provided $I(x, y)=0$ if $(x, y) \notin \Omega$. For practical implementation, we usually set

$$
k_{p, q}=\frac{1}{K^{2}}, \quad \forall p, q=1, \ldots, K
$$

with an appropriate choice of $K \in \mathbb{N}$.

Assumption 3. The spectral energy $Y_{I}$ of the retrieved high resolution multispectral image $I: \Omega \rightarrow \mathbb{R}^{4}$ is an element of the Sobolev space with variable exponent $W^{1, p(\cdot)}(\Omega)$, where $p(\cdot)$ is defined in $(4.2)$, and

$$
Y_{I}(x)=\alpha_{R} I_{R}(x)+\alpha_{G} I_{G}(x)+\alpha_{B} I_{B}(x), \quad \forall x \in \Omega
$$

with $\alpha_{R}=0.299, \alpha_{G}=0.587$, and $\alpha_{B}=0.114$.

Assumption 4. The topographic maps for each spectral channels $I_{R}, I_{G}, I_{B}$, and $I_{N I R}$ of the retrieved image $I: \Omega \rightarrow \mathbb{R}^{4}$ have a similar structure to the topographic map of the spectral energy $Y_{H}$ of the Landsat image $H: \Omega \rightarrow$ $\mathbb{R}^{3}$.

As follows from this Assumption, all spectral channels of the retrieved image should share the geometry of the panchromatic image $Y_{H}$ in $\Omega$. It means that, due to the property $Y_{H} \in B V(\Omega)$, for almost all points of almost all level sets of $Y_{H}$ we can define a normal vector $\theta(x)$, i.e., it formally satisfies $\left(\theta, Y_{H}\right)=\left|\nabla Y_{H}\right|$ and $|\theta| \leqslant 1$ a.e. in $\Omega$ (see Remark 3.1 for the details). So, if $\theta \in L^{\infty}\left(\Omega, \mathbb{R}^{2}\right)$ is a vector field with indicated properties, it follows that $\theta(x)$ has the direction of the normal to the level lines of $Y_{H}$. Therefore, the counterclockwise rotation of angle $\pi / 2$, denoted by $\theta^{\perp}$, represents the tangent vector to the level lines of $Y_{H}$. In this case, if the spectral channels of $I: \Omega \rightarrow \mathbb{R}^{4}$ share the geometry of the panchromatic image $Y_{H}$, we have

$$
\left(\theta^{\perp}, \nabla I_{i}\right)_{\mathbb{R}^{2}}=0, \quad i \in\{R, G, B, N I R\} \text { in } \Omega .
$$

\section{Statement of the Spatial Interpolation Problem}

The problem of spatial interpolation of the MODIS-like image $L: S_{L} \rightarrow \mathbb{R}^{4}$ to the resolution of three-band Landsat-like image $H: S_{H} \rightarrow \mathbb{R}^{3}$ consists in the 
restoration of the four-band image $I: \Omega \rightarrow \mathbb{R}^{4}$ such that properties (i)-(v) would be satisfied. To do so, we propose at the first stage to compute the high resolution images $I_{R}, I_{G}, I_{B}: \Omega \rightarrow \mathbb{R}$ as a solution of the following constrained minimization problem

$$
\inf _{\left(I_{R}, I_{G}, I_{B}\right) \in \Xi} \mathcal{J}\left(I_{R}, I_{G}, I_{B}\right),
$$

where $\Xi$ denotes the set of admissible images, and $\mathcal{J}\left(I_{R}, I_{G}, I_{B}\right)$ stands for the energy functional. Here, we define the set $\Xi$ as follows: $\left(I_{R}, I_{G}, I_{B}\right) \in \Xi$ if and only if

(A) $\left(I_{R}, I_{G}, I_{B}\right) \in W^{1, p(\cdot)}\left(\Omega ; \mathbb{R}^{3}\right)$, where $p(\cdot)$ stands for the texturity characteristic of the spectral energy $Y_{H} \in B V(\Omega)$;

(B) the following pointwise inequalities

$$
\begin{aligned}
& 0 \leqslant I_{R}(x, y) \leqslant \max _{\left(x_{i}, y_{j}\right) \in S_{L}} L_{R}\left(x_{i}, y_{j}\right) \text { a.e. in } \Omega, \\
& 0 \leqslant I_{G}(x, y) \leqslant \max _{\left(x_{i}, y_{j}\right) \in S_{L}} L_{G}\left(x_{i}, y_{j}\right) \text { a.e. in } \Omega, \\
& 0 \leqslant I_{B}(x, y) \leqslant \max _{\left(x_{i}, y_{j}\right) \in S_{L}} L_{B}\left(x_{i}, y_{j}\right) \text { a.e. in } \Omega .
\end{aligned}
$$

hold true.

As for the energy functional $\mathcal{J}: \Xi \rightarrow \mathbb{R}$, we construct it in the form

$$
\mathcal{J}=\mathcal{J}_{0}+\gamma \mathcal{J}_{1}+\lambda \mathcal{J}_{2}+\mu \mathcal{J}_{3}
$$

where

$$
\begin{aligned}
\mathcal{J}_{0}\left(I_{R}, I_{G}, I_{B}\right)= & \int_{\Omega} \frac{1}{p(x)}\left|\nabla I_{R}(x)\right|^{p(x)} d x+\int_{\Omega} \frac{1}{p(x)}\left|\nabla I_{G}(x)\right|^{p(x)} d x \\
& +\int_{\Omega} \frac{1}{p(x)}\left|\nabla I_{B}(x)\right|^{p(x)} d x \\
\mathcal{J}_{1}\left(I_{R}, I_{G}, I_{B}\right)= & \int_{\Omega}\left|\left(\theta^{\perp}, \nabla I_{R}\right)\right|^{\alpha} d x+\int_{\Omega}\left|\left(\theta^{\perp}, \nabla I_{G}\right)\right|^{\alpha} d x \\
& +\int_{\Omega}\left|\left(\theta^{\perp}, \nabla I_{B}\right)\right|^{\alpha} d x, \\
\mathcal{J}_{2}\left(I_{R}, I_{G}, I_{B}\right)= & \int_{\Omega}\left[\alpha_{R} I_{R}+\alpha_{G} I_{G}+\alpha_{B} I_{B}-Y_{H}\right]^{2} d x, \\
\mathcal{J}_{3}\left(I_{R}, I_{G}, I_{B}\right)= & \sum_{i=1}^{M_{x}} \sum_{j=1}^{M_{y}}\left(\left[\mathcal{K} * I_{R}\right]\left(x_{i}, y_{j}\right)-L_{R}\left(x_{i}, y_{j}\right)\right)^{2} \\
& +\sum_{i=1}^{M_{x}} \sum_{j=1}^{M_{y}}\left(\left[\mathcal{K} * I_{G}\right]\left(x_{i}, y_{j}\right)-L_{G}\left(x_{i}, y_{j}\right)\right)^{2} \\
& +\sum_{i=1}^{M_{x}} \sum_{j=1}^{M_{y}}\left(\left[\mathcal{K} * I_{B}\right]\left(x_{i}, y_{j}\right)-L_{B}\left(x_{i}, y_{j}\right)\right)^{2},
\end{aligned}
$$


and

$$
\theta(x, y)=\frac{D U(t, x, y)}{|D U(t, x, y)|} \text { for small values of } t>0 .
$$

Here, the exponent $\alpha>0$ is defined by (4.6), and $U(t, x, y)$ is the solution of the parabolic problem (3.2)-(3.4) with the initial condition

$$
U(0, x, y)=Y_{H}(x, y)=\alpha_{R} H_{R}(x, y)+\alpha_{G} H_{G}(x, y)+\alpha_{B} H_{B}(x, y), \quad \forall(x, y) \in \Omega .
$$

The main motivation for such choice of the energy functional is rather clear. As follows from (5.9), each term in $\mathcal{J}_{3}\left(I_{R}, I_{G}, I_{B}\right)$ represents an $L^{2}$-distortion between a particular spectral channel in the MODIS image $L: S_{L} \rightarrow \mathbb{R}^{4}$ and the corresponding channel of the retrieved image $I: S_{H} \rightarrow \mathbb{R}^{4}$ which is resampled to the grid of low resolution $S_{L}$. So, the $\mathcal{J}_{3}$-term should be minimal and it is mainly motivated by Assumption 2 .

As for the term $\mathcal{J}_{2}\left(I_{R}, I_{G}, I_{B}\right)$, it reflects the fact that the spectral energy $Y_{I}=\alpha_{R} I_{R}+\alpha_{G} I_{G}+\alpha_{B} I_{B}$ of the retrieved image should be as close as possible to the spectral energy of the Landsat image $H: S_{H} \rightarrow \mathbb{R}^{3}$. We interpret this closedness in the sense of $L^{2}$-norm.

Now about the term $\mathcal{J}_{1}\left(I_{R}, I_{G}, I_{B}\right)$. As was mentioned before, the main goal, we are going to follows in the spatial interpolation problem, is to preserve the following property: the geometry of each spectral channels in the retrieved image should be as close as possible to the geometry of the spectral energy of the Landsat image $H: S_{H} \rightarrow \mathbb{R}^{3}$. Formally, it means that the following relations have to be satisfied

$$
\left(\theta^{\perp}, \nabla I_{R}\right)_{\mathbb{R}^{2}}=0, \quad\left(\theta^{\perp}, \nabla I_{G}\right)_{\mathbb{R}^{2}}=0, \quad\left(\theta^{\perp}, \nabla I_{B}\right)_{\mathbb{R}^{2}}=0 \quad \text { a.e. in } \Omega .
$$

Hence, the magnitude

$$
\int_{\Omega}\left[\left|\left(\theta^{\perp}, \nabla I_{R}\right)\right|^{\alpha}+\left|\left(\theta^{\perp}, \nabla I_{G}\right)\right|^{\alpha}+\left|\left(\theta^{\perp}, \nabla I_{B}\right)\right|^{\alpha}\right] d x
$$

must be small enough, where $\theta=\theta(x, y)$ stands for the vector field of unit normals to the topographic map of the spectral energy $Y_{H}=\alpha_{R} H_{R}+\alpha_{G} H_{G}+\alpha_{B} H_{B}$.

The first term $\mathcal{J}_{0}\left(I_{R}, I_{G}, I_{B}\right)$ is the regularization. Since $p(x) \approx 1$ in places in $\Omega$ where edges or discontinuities are present in the spectral energy $Y_{H}$ of the image $H$, and $p(x) \approx 2$ in places where $Y_{H}(x)$ is smooth or contains homogeneous features, the main benefit of the model (5.1) is the manner in which it accommodates the local image information. The places where the gradient is sufficiently large (i.e. likely edges), we deal with the so-called TV-based diffusion [18], whereas the places where the gradient is close to zero (i.e. homogeneous regions), the model becomes isotropic. Specifically, the type of anisotropy at these ambiguous regions varies according to the strength of the gradient. This enables the model to have a much lower dependence on the approximation schemes for the variable exponent $p(x)$ and other thresholds. 
We are now in a position to define what we mean by the solution of spatial interpolation problem that was stated in Section 2. Taking into account the properties (i)-(v) that we imposed and the structure of the energy functional $\mathcal{J}: \Xi \rightarrow \mathbb{R}$, we say that a four-band image $I^{0}=\left[I_{R}^{0}, I_{G}^{0}, I_{B}^{0}, I_{N I R}^{0}\right]^{t}: S_{H} \rightarrow \mathbb{R}^{4}$ is the result of fusion of MODIS-like multi spectral image $L: S_{L} \rightarrow \mathbb{R}^{4}$ with the Landsat-like color image $H: S_{H} \rightarrow \mathbb{R}^{3}$ at higher resolution if:

- the triplet $\left(I_{R}^{0}, I_{G}^{0}, I_{B}^{0}\right)$ is a solution of constrained minimization problem (5.1), i.e.,

$$
\left(I_{R}^{0}, I_{G}^{0}, I_{B}^{0}\right) \in \Xi \text { and } \mathcal{J}\left(I_{R}^{0}, I_{G}^{0}, I_{B}^{0}\right)=\inf _{\left(I_{R}, I_{G}, I_{B}\right) \in \Xi} \mathcal{J}\left(I_{R}, I_{G}, I_{B}\right)
$$

- The spectral channel $I_{N I R}^{0}: \Omega \rightarrow \mathbb{R}$ is defined as follows

$$
I_{N I R}^{0}(x, y)=\gamma_{R} I_{R}^{0}(x, y)+\gamma_{G} I_{G}^{0}(x, y)+\gamma_{B} I_{B}^{0}((x, y), \quad \forall(x, y) \in \Omega,
$$

where

$$
\left[\begin{array}{c}
\gamma_{R} \\
\gamma_{G} \\
\gamma_{B}
\end{array}\right]=\left[\int_{\Omega}\left(\begin{array}{ccc}
L_{R}^{2} & L_{R} L_{G} & L_{R} L_{B} \\
L_{R} L_{G} & L_{R}^{2} & L_{G} L_{B} \\
L_{R} L_{B} & L_{G} L_{B} & L_{B}^{2}
\end{array}\right) d x\right]^{-1} \int_{\Omega}\left[\begin{array}{c}
L_{N I R} L_{R} \\
L_{N I R} L_{G} \\
L_{N I R} L_{B}
\end{array}\right] d x
$$

Here, the last equality is a formal representation of the solution to the following linear regression problem

$$
\int_{\Omega}\left[\gamma_{R} L_{R}+\gamma_{G} L_{G}+\gamma_{B} L_{B}-L_{N I R}\right]^{2} d x \stackrel{\gamma_{R}, \gamma_{G} \gamma_{B}}{\longrightarrow} \inf .
$$

Remark 5.1. As an alternative way to define the NIR spectral channel $I_{N I R}^{0}$, we can propose the following one: define $I_{N I R}^{0}$ as a solution of the constrained minimization problem

$$
\mathcal{Z}\left(I_{N I R}^{0}\right)=\inf _{I \in \Xi_{N I R}} \mathcal{Z}(I)
$$

where

$$
\begin{aligned}
\mathcal{Z}(I)= & \int_{\Omega} \frac{1}{p(x)}|\nabla I(x)|^{p(x)} d x+\gamma \int_{\Omega}\left|\left(\theta^{\perp}, \nabla I\right)\right|^{\alpha} d x \\
& \quad+\lambda \int_{\Omega}\left[\gamma_{R} I_{R}^{0}+\gamma_{G} I_{G}^{0}+\gamma_{B} I_{B}^{0}-I\right]^{2} d x \\
\Xi_{N I R}= & \left\{I \in W^{1, p(\cdot)}(\Omega): 0 \leqslant I(x, y) \leqslant \max _{\left(x_{i}, y_{j}\right) \in S_{L}} L_{N I R}\left(x_{i}, y_{j}\right) \text { a.e. in } \Omega\right\},
\end{aligned}
$$

and the weight coefficients $\gamma_{R}, \gamma_{G}, \gamma_{B}$ are defined by (5.11). 
Remark 5.2. Another variant for the setting of the spatial interpolation problem is to consider, instead of the energy term $\mathcal{J}_{1}$ in (5.5), the following functional (this approach was firstly proposed in [5])

$$
\begin{gathered}
\mathcal{J}_{1}\left(I_{R}, I_{G}, I_{B}\right)=\left(\int_{\Omega}\left|\nabla I_{R}\right| d x+\int_{\Omega} I_{R} \operatorname{div} \theta d x\right) \\
+\left(\int_{\Omega}\left|\nabla I_{G}\right| d x+\int_{\Omega} I_{G} \operatorname{div} \theta d x\right) \\
\quad+\left(\int_{\Omega}\left|\nabla I_{B}\right| d x+\int_{\Omega} I_{B} \operatorname{div} \theta d x\right) .
\end{gathered}
$$

The main motivation for such choice of the functional $\mathcal{J}_{1}$ is rather clear. Since the geometry of each spectral channel in the retrieved image should be as close as possible to the geometry of the spectral energy of the Landsat image $H: S_{H} \rightarrow \mathbb{R}^{3}$, it means that the following relations have to be satisfied

$$
\left|\nabla I_{R}\right|=\left(\theta, \nabla I_{R}\right),\left|\nabla I_{G}\right|=\left(\theta, \nabla I_{G}\right),\left|\nabla I_{B}\right|=\left(\theta, \nabla I_{B}\right), \text { a.e. in } \Omega .
$$

Hence, the magnitude

$$
\int_{\Omega}\left[\left(\left|\nabla I_{R}\right|-\left(\theta, \nabla I_{R}\right)\right)+\left(\left|\nabla I_{G}\right|-\left(\theta, \nabla I_{G}\right)\right)+\left(\left|\nabla I_{B}\right|-\left(\theta, \nabla I_{B}\right)\right)\right] d x
$$

must be small enough, where $\theta=\theta(x, y)$ stands for the vector field of unit normals to the topographic map of the spectral energy $Y_{H}=\alpha_{R} H_{R}+\alpha_{G} H_{G}+\alpha_{B} H_{B}$. By default, we assume that this field is zero along the boundary $\partial \Omega$. Then, making use of the Green's formula, we deduce:

$$
\begin{aligned}
& \int_{\Omega}\left(\left|\nabla I_{R}\right|-\theta \cdot \nabla I_{R}\right) d x=\int_{\Omega}\left|\nabla I_{R}\right| d x+\int_{\Omega} I_{R} \operatorname{div} \theta d x, \\
& \int_{\Omega}\left(\left|\nabla I_{G}\right|-\theta \cdot \nabla I_{G}\right) d x=\int_{\Omega}\left|\nabla I_{B}\right| d x+\int_{\Omega} I_{B} \operatorname{div} \theta d x, \\
& \int_{\Omega}\left(\left|\nabla I_{B}\right|-\theta \cdot \nabla I_{B}\right) d x=\int_{\Omega}\left|\nabla I_{B}\right| d x+\int_{\Omega} I_{B} \operatorname{div} \theta d x .
\end{aligned}
$$

\section{Existence Result and Optimality Conditions}

\subsection{On Existence and Uniqueness of Retrieved Image at High Resolution}

We begin this section with the following existence result.

Theorem 6.1. Let $H: S_{H} \rightarrow \mathbb{R}^{3}$ and $L: S_{L} \rightarrow \mathbb{R}^{4}$ be given images such that their spectral energies satisfy conditions (4.1). Then, under Assumptions 1-4, there exists a unique triplet $\left(I_{R}, I_{G}, I_{B}\right) \in \Xi \subset B V\left(\Omega ; \mathbb{R}^{3}\right)$ such that $\left(I_{R}, I_{G}, I_{B}\right)$ is a minimizer to constrained minimization problem (5.1). 
Proof. First of all, we notice that minimization problem (5.1) is consistent, moreover, $\mathcal{J}\left(I_{R}, I_{G}, I_{B}\right)<+\infty$ for each feasible triplet $\left(I_{R}, I_{G}, I_{B}\right) \in \Xi$. Indeed, in view of the pointwise estimates (5.2)-(5.4), we have $I_{R}, I G, I_{B} \in L^{2}(\Omega)$. Hence,

$$
\mathcal{J}_{2}\left(I_{R}, I_{G}, I_{B}\right)<+\infty \text {. }
$$

It remains to notice that the inclusion $I \in W^{1, p(\cdot)}\left(\Omega ; \mathbb{R}^{4}\right)$ and the estimates

$$
\begin{aligned}
\int_{\Omega}\left|\left(\theta^{\perp}, \nabla I_{A}\right)\right|^{\alpha} d x & \leqslant\|\theta\|_{L^{\infty}\left(\Omega ; \mathbb{R}^{2}\right)}^{\alpha}\left\|\nabla I_{A}\right\|_{L^{\alpha}\left(\Omega ; \mathbb{R}^{2}\right)}^{\alpha} \\
& \text { by }(3.13)-(3.15) \\
& \leqslant(1+|\Omega|)\|\theta\|_{L^{\infty}\left(\Omega ; \mathbb{R}^{2}\right)}^{\alpha}\left\|I_{A}\right\|_{W^{1, p(\cdot)}\left(\Omega ; \mathbb{R}^{2}\right)}, \quad A \in\{R, G, B\}
\end{aligned}
$$

imply $\mathcal{J}_{1}\left(I_{R}, I_{G}, I_{B}\right)<+\infty$. As a result, consistency of the problem (5.1) immediately follows from (5.5)-(5.9) and definition of the set $\Xi$.

Let $\left\{\left(I_{R}^{k}, I_{G}^{k}, I_{B}^{k}\right)\right\}_{k \in \mathbb{N}} \subset \Xi$ be a minimizing sequence to the problem (5.1). i.e.,

$$
\lim _{k \rightarrow \infty} \mathcal{J}\left(I_{R}^{k}, I_{G}^{k}, I_{B}^{k}\right)=\inf _{\left(I_{R}, I_{G}, I_{B}\right) \in \Xi} \mathcal{J}\left(I_{R}, I_{G}, I_{B}\right) .
$$

Then there exists a constant $\widehat{C}>0$ such that

$$
\sup _{k \in \mathbb{N}} \mathcal{J}\left(I_{R}^{k}, I_{G}^{k}, I_{B}^{k}\right) \leqslant \widehat{C} .
$$

From this and (5.5), we deduce that

$$
\begin{gathered}
\int_{\Omega} \frac{1}{p(x)}\left[\left|\nabla I_{R}(x)\right|^{p(x)}+\left|\nabla I_{G}(x)\right|^{p(x)}+\left|\nabla I_{B}(x)\right|^{p(x)}\right] d x \leqslant \widehat{C}, \\
\int_{\Omega}\left[\alpha_{R} I_{R}^{k}+\alpha_{G} I_{G}^{k}+\alpha_{B} I_{B}^{k}-Y_{H}\right]^{2} d x \leqslant \widehat{C} .
\end{gathered}
$$

Since $\alpha:=1+\delta \leqslant p(x) \leqslant \beta:=2$ for all $x \in \Omega$, it follows from (6.1) and (3.11) that

$$
\sup _{k \in \mathbb{N}}\left[\left\|\nabla I_{R}\right\|_{L^{p(\cdot)}\left(\Omega ; \mathbb{R}^{2}\right)}^{\alpha}+\left\|\nabla I_{G}\right\|_{L^{p(\cdot)}\left(\Omega ; \mathbb{R}^{2}\right)}^{\alpha}+\left\|\nabla I_{B}\right\|_{L^{p(\cdot)}\left(\Omega ; \mathbb{R}^{2}\right)}^{\alpha}\right] \leqslant \alpha(\widehat{C}+3) .
$$

On th other hand, utilizing estimate (6.2) and non-negativity of $I_{R}^{k}, I_{G}^{k}, I_{B}^{k}$, and $Y_{H}$, we obtain

$$
\int_{\Omega}\left[\alpha_{R} I_{R}^{k}+\alpha_{G} I_{G}^{k}+\alpha_{B} I_{B}^{k}\right]^{2} d x \leqslant 2 \widehat{C}+2 \int_{\Omega} Y_{H}^{2} d x
$$

Hence,

$$
\begin{array}{ll}
(1+|\Omega|)^{-1} \sup _{k \in \mathbb{N}}\left\|I_{R}^{k}\right\|_{L^{p(\cdot)}(\Omega)}^{2} & \stackrel{\text { by }(3.14)}{\leqslant} \sup _{k \in \mathbb{N}}\left\|I_{R}^{k}\right\|_{L^{2}(\Omega)}^{2} \leqslant 2 \alpha_{R}^{-2}\left[\widehat{C}+\left\|Y_{H}\right\|_{L^{2}(\Omega)}^{2}\right], \\
(1+|\Omega|)^{-1} \sup _{k \in \mathbb{N}}\left\|I_{G}^{k}\right\|_{L^{p(\cdot)}(\Omega)}^{2} & \stackrel{\text { by }(3.14)}{\leqslant} \sup _{k \in \mathbb{N}}\left\|I_{G}^{k}\right\|_{L^{2}(\Omega)}^{2} \leqslant 2 \alpha_{G}^{-2}\left[\widehat{C}+\left\|Y_{H}\right\|_{L^{2}(\Omega)}^{2}\right], \\
(1+|\Omega|)^{-1} \sup _{k \in \mathbb{N}}\left\|I_{B}^{k}\right\|_{L^{p(\cdot)}(\Omega)}^{2} & \stackrel{\text { by (3.14) }}{\leqslant} \sup _{k \in \mathbb{N}}\left\|I_{B}^{k}\right\|_{L^{2}(\Omega)}^{2} \leqslant 2 \alpha_{B}^{-2}\left[\widehat{C}+\left\|Y_{H}\right\|_{L^{2}(\Omega)}^{2}\right] .
\end{array}
$$


As a result, it follows from (6.3) and (6.4) that

$$
\begin{aligned}
& \sup _{k \in \mathbb{N}}\left\|I_{R}^{k}\right\|_{W^{1, p(\cdot)}(\Omega)}=\sup _{k \in \mathbb{N}}\left[\left\|I_{R}^{k}\right\|_{L^{p(\cdot)}(\Omega)}+\left\|\nabla I_{R}^{k}\right\|_{L^{p(\cdot)}\left(\Omega ; \mathbb{R}^{2}\right)}\right] \leqslant \mathcal{Q}_{R}, \\
& \sup _{k \in \mathbb{N}}\left\|I_{G}^{k}\right\|_{W^{1, p(\cdot)}(\Omega)}=\sup _{k \in \mathbb{N}}\left[\left\|I_{G}^{k}\right\|_{L^{p(\cdot)}(\Omega)}+\left\|\nabla I_{G}^{k}\right\|_{L^{p(\cdot)}\left(\Omega ; \mathbb{R}^{2}\right)}\right] \leqslant \mathcal{Q}_{G}, \\
& \sup _{k \in \mathbb{N}}\left\|I_{B}^{k}\right\|_{W^{1, p(\cdot)}(\Omega)}=\sup _{k \in \mathbb{N}}\left[\left\|I_{B}^{k}\right\|_{L^{p(\cdot)}(\Omega)}+\left\|\nabla I_{B}^{k}\right\|_{L^{p(\cdot)}\left(\Omega ; \mathbb{R}^{2}\right)}\right] \leqslant \mathcal{Q}_{B}
\end{aligned}
$$

with $\mathcal{Q}_{A}=\left[2(1+|\Omega|) \alpha_{A}^{-2}\left(\widehat{C}+\left\|Y_{H}\right\|_{L^{2}(\Omega)}^{2}\right)\right]^{1 / 2}+[\alpha(\widehat{C}+3)]^{1 / \alpha}, A \in\{R, G, B\}$.

Thus, the sequence $\left\{\left(I_{R}^{k}, I_{G}^{k}, I_{B}^{k}\right)\right\}_{k \in \mathbb{N}} \subset \Xi$ is bounded in $W^{1, p(\cdot)}\left(\Omega ; \mathbb{R}^{3}\right)$. Therefore, in view of Proposition 3.3, there exists a subsequence of

$$
\left\{\left(I_{R}^{k}, I_{G}^{k}, I_{B}^{k}\right)\right\}_{k \in \mathbb{N}} \subset \Xi
$$

still denoted by the same index, and functions $\left(I_{R}^{0}, I_{G}^{0}, I_{B}^{0}\right) \in W^{1, p(\cdot)}\left(\Omega ; \mathbb{R}^{3}\right)$ such that

$$
\begin{gathered}
\left(I_{R}^{k}, I_{G}^{k}, I_{B}^{k}\right) \rightarrow\left(I_{R}^{0}, I_{G}^{0}, I_{B}^{0}\right) \text { weakly in } W^{1, p(\cdot)}\left(\Omega ; \mathbb{R}^{3}\right), \\
\left(I_{R}^{k}, I_{G}^{k}, I_{B}^{k}\right) \rightarrow\left(I_{R}^{0}, I_{G}^{0}, I_{B}^{0}\right) \text { weakly in } W^{1, \alpha}\left(\Omega ; \mathbb{R}^{3}\right), \\
\left(I_{R}^{k}, I_{G}^{k}, I_{B}^{k}\right) \rightarrow\left(I_{R}^{0}, I_{G}^{0}, I_{B}^{0}\right) \text { strongly in } L^{\alpha}\left(\Omega ; \mathbb{R}^{3}\right),
\end{gathered}
$$

and

$$
\begin{aligned}
& \int_{\Omega} \frac{1}{p(x)}\left|\nabla I_{R}^{0}\right|^{p(x)} d x \leqslant \liminf _{k \rightarrow \infty} \int_{\Omega} \frac{1}{p(x)}\left|\nabla I_{R}^{k}\right|^{p(x)} d x, \\
& \int_{\Omega} \frac{1}{p(x)}\left|\nabla I_{G}^{0}\right|^{p(x)} d x \leqslant \liminf _{k \rightarrow \infty} \int_{\Omega} \frac{1}{p(x)}\left|\nabla I_{G}^{k}\right|^{p(x)} d x, \\
& \int_{\Omega} \frac{1}{p(x)}\left|\nabla I_{B}^{0}\right|^{p(x)} d x \leqslant \liminf _{k \rightarrow \infty} \int_{\Omega} \frac{1}{p(x)}\left|\nabla I_{B}^{k}\right|^{p(x)} d x .
\end{aligned}
$$

Moreover, passing to a subsequence if necessary and taking into account the inequalities (5.2)-(5.4) and (3.13), we have:

$$
\begin{gathered}
\left(I_{R}^{k}(x, y), I_{G}^{k}(x), I_{B}^{k}(x)\right) \rightarrow\left(I_{R}^{0}(x), I_{G}^{0}(x), I_{B}^{0}(x)\right) \text { for a.e. } x \in \Omega, \\
\left(I_{R}^{k}, I_{G}^{k}, I_{B}^{k}\right) \rightarrow\left(I_{R}^{0}, I_{G}^{0}, I_{B}^{0}\right) \text { weakly in } L^{2}\left(\Omega ; \mathbb{R}^{3}\right), \\
\left(\theta^{\perp}, \nabla I_{A}^{k}\right) \rightarrow\left(\theta^{\perp}, \nabla I_{A}^{0}\right) \quad \text { weakly in } L^{\alpha}(\Omega) \text { for } A \in\{R, G, B\} .
\end{gathered}
$$

Hence, without loss of generality, we can suppose that the limit triplet $\left(I_{R}^{0}, I_{G}^{0}, I_{B}^{0}\right)$ satisfies the pointwise restrictions (5.2)-(5.4), and, as a consequence, we deduce: $\left(I_{R}^{0}, I_{G}^{0}, I_{B}^{0}\right) \in \Xi$ is a feasible solution to the problem (5.1). 
Let us show that $\left(I_{R}^{0}, I_{G}^{0}, I_{B}^{0}\right) \in \Xi$ is a minimizer to the problem (5.1). Indeed, utilizing convergence properties (6.5)-(6.12), we get

$$
\begin{aligned}
& \liminf _{k \rightarrow \infty} \mathcal{J}_{0}\left(I_{R}^{k}, I_{G}^{k}, I_{B}^{k}\right) \stackrel{\text { by }(6.8)-(6.10)}{\geqslant} \mathcal{J}_{0}\left(I_{R}^{0}, I_{G}^{0}, I_{B}^{0}\right), \\
& \liminf _{k \rightarrow \infty} \mathcal{J}_{1}\left(I_{R}^{k}, I_{G}^{k}, I_{B}^{k}\right) \stackrel{\text { by }}{\stackrel{(6.13)}{\geqslant}} \mathcal{J}_{1}\left(I_{R}^{0}, I_{G}^{0}, I_{B}^{0}\right), \\
& \liminf _{k \rightarrow \infty} \mathcal{J}_{2}\left(I_{R}^{k}, I_{G}^{k}, I_{B}^{k}\right) \stackrel{\text { by }}{\stackrel{(6.12)}{\geqslant}} \mathcal{J}_{2}\left(I_{R}^{0}, I_{G}^{0}, I_{B}^{0}\right), \\
& \liminf _{k \rightarrow \infty} \mathcal{J}_{3}\left(I_{R}^{k}, I_{G}^{k}, I_{B}^{k}\right) \stackrel{\text { by }(6.11),(6.12)}{\geqslant} \mathcal{J}_{3}\left(I_{R}^{0}, I_{G}^{0}, I_{B}^{0}\right) \text {. }
\end{aligned}
$$

Hence, $\liminf _{k \rightarrow \infty} \mathcal{J}\left(I_{R}^{k}, I_{G}^{k}, I_{B}^{k}\right) \geqslant \mathcal{J}\left(I_{R}^{0}, I_{G}^{0}, I_{B}^{0}\right)$, and, therefore,

$$
\begin{aligned}
\inf _{\left(I_{R}, I_{G}, I_{B}\right) \in \Xi} \mathcal{J}\left(I_{R}, I_{G}, I_{B}\right) & =\lim _{k \rightarrow \infty} \mathcal{J}\left(I_{R}^{k}, I_{G}^{k}, I_{B}^{k}\right)=\liminf _{k \rightarrow \infty} \mathcal{J}\left(I_{R}^{k}, I_{G}^{k}, I_{B}^{k}\right) \\
& \geqslant \mathcal{J}\left(I_{R}^{0}, I_{G}^{0}, I_{B}^{0}\right) \geqslant \inf _{\left(I_{R}, I_{G}, I_{B}\right) \in \Xi} \mathcal{J}\left(I_{R}, I_{G}, I_{B}\right) .
\end{aligned}
$$

Thus, $\left(I_{R}^{0}, I_{G}^{0}, I_{B}^{0}\right)$ is a minimiser to the problem (5.1).

It remains to show that $\left(I_{R}^{0}, I_{G}^{0}, I_{B}^{0}\right)$ is a unique minimizer for this problem. Indeed, let us assume the converse. Let $\left(I_{R}^{0}, I_{G}^{0}, I_{B}^{0}\right) \in \Xi$ and $\left(I_{R}^{*}, I_{G}^{*}, I_{B}^{*}\right) \in \Xi$ be two minimizers for the problem (5.1). Then by the strict convexity of norm $\|\cdot\|_{L^{2}(\Omega)}$ and convexity of the set of feasible solutions $\Xi$, we have

$$
\begin{aligned}
\mathcal{J}\left(\frac{I_{R}^{0}+I_{R}^{*}}{2}, \frac{I_{G}^{0}+I_{G}^{*}}{2}, \frac{I_{B}^{0}+I_{B}^{*}}{2}\right) & <\frac{1}{2} \mathcal{J}\left(I_{R}^{0}, I_{G}^{0}, I_{B}^{0}\right)+\frac{1}{2} \mathcal{J}\left(I_{R}^{*}, I_{G}^{*}, I_{B}^{*}\right) \\
& =\inf _{\left(I_{R}, I_{G}, I_{B}\right) \in \Xi} \mathcal{J}\left(I_{R}, I_{G}, I_{B}\right)
\end{aligned}
$$

which brings us into a conflict with the initial assumptions. Thus, $\left(I_{R}^{0}, I_{G}^{0}, I_{B}^{0}\right)$ is a unique minimizer to the problem (5.1). The proof is complete.

For further convenience, we rewrite the energy functional $\mathcal{J}: \Xi \rightarrow \mathbb{R}$ in the integral form. To this end, we set: let $\delta_{(i, j)}$ be the Dirac's delta on the point $(i . j) \in \Omega$. Let $\Pi_{L}=\sum_{(i, j) \in S_{L}} \delta_{(i, j)}$ be the Dirac's comb on $\Omega$ defined by the grid $S_{L}$. Then we may write $\mathcal{J}_{3}$ in integral terms

$$
\mathcal{J}_{3}\left(I_{R}, I_{G}, I_{B}\right)=\sum_{A \in\{R, G . B\}} \int_{\Omega} \Pi_{L}\left(\left[\mathcal{K} * I_{A}\right](x)-L_{A}(x)\right)^{2} d x,
$$

where $L_{A}, A \in\{R, G . B\}$ denotes an arbitrary extension of $L_{A}(i, j)$ as continuous functions from $S_{L}$ to $\Omega$. Since the terms above are multiplied by $\Pi_{L}$, the integral terms in (6.14) do not depend on the particular extensions of $L_{A}, A \in\{R, G . B\}$. 


\subsection{Optimality Conditions}

In order to derive some optimality conditions to the problem (5.1) and characterize its solution $\left(I_{R}^{0}, I_{G}^{0}, I_{B}^{0}\right)$, we check that the functional $\mathcal{J}: \Xi \rightarrow \mathbb{R}$ is Gâteaux differentiable. To this end, we note that

$$
\begin{aligned}
& \frac{\left|\nabla I_{A}^{0}(x)+t \nabla h_{A}(x)\right|^{p(x)}-\left|\nabla I_{A}^{0}(x)\right|^{p(x)}}{p(x) t} \\
& \quad \rightarrow\left(\left|\nabla I_{A}^{0}(x)\right|^{p(x)-2} \nabla I_{A}^{0}(x), \nabla h_{A}(x)\right) \text { as } t \rightarrow 0
\end{aligned}
$$

almost everywhere in $\Omega$ for all $A \in\{R, G . B\}$ and $h=\left(h_{R}, h_{G}, h_{B}\right) \in W^{1, p(\cdot)}(\Omega)^{3}$. Indeed, by convexity, we have $|\xi|^{p}-|\eta|^{p} \leqslant 2 p\left(|\xi|^{p-1}+|\eta|^{p-1}\right)|\xi-\eta|$. Then

$$
\begin{aligned}
& \left|\frac{\left|\nabla I_{A}^{0}(x)+t \nabla h_{A}(x)\right|^{p(x)}-\left|\nabla I_{A}^{0}(x)\right|^{p(x)}}{p(x) t}\right| \\
& \leqslant 2\left(\left|\nabla I_{A}^{0}(x)+t \nabla h_{A}(x)\right|^{p(x)-1}+\left|\nabla I_{A}^{0}(x)\right|^{p(x)-1}\right)\left|\nabla h_{A}(x)\right| \\
& \leqslant \operatorname{const}\left(\left|\nabla I_{A}^{0}(x)\right|^{p(x)-1}+\left|\nabla h_{A}(x)\right|^{p(x)-1}\right)\left|\nabla h_{A}(x)\right| .
\end{aligned}
$$

Taking into account that

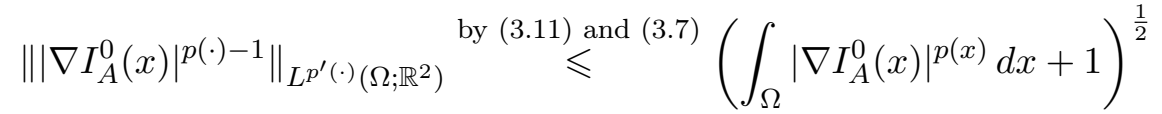

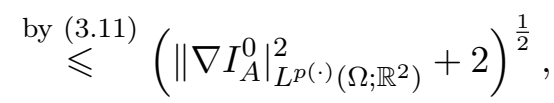

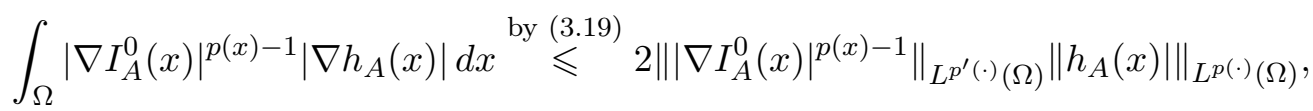

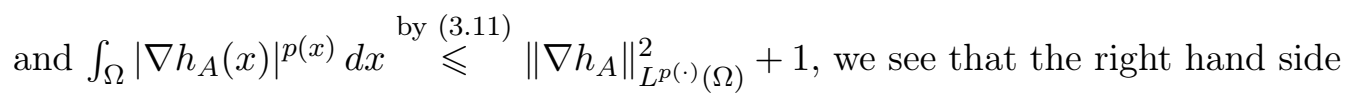
of inequality (6.15) is an $L^{1}(\Omega)$ function. Therefore,

$$
\begin{aligned}
& \int_{\Omega} \frac{\left|\nabla I_{A}^{0}(x)+t \nabla h_{A}(x)\right|^{p(x)}-\left|\nabla I_{A}^{0}(x)\right|^{p(x)}}{p(x) t} d x \\
& \rightarrow \int_{\Omega}\left(\left|\nabla I_{A}^{0}(x)\right|^{p(x)-2} \nabla I_{A}^{0}(x), \nabla h_{A}(x)\right) d x \text { as } t \rightarrow 0
\end{aligned}
$$

by the Lebesgue dominated convergence theorem for each $h=\left(h_{R}, h_{G}, h_{B}\right) \in$ 
$W^{1, p(\cdot)}\left(\Omega ; \mathbb{R}^{3}\right)$ and $A \in\{R, G . B\}$. Thus,

$$
\begin{aligned}
& \lim _{t \rightarrow 0} \frac{\mathcal{J}_{0}\left(I_{R}^{0}+t h_{R}, I_{G}^{0}+t h_{G}, I_{B}^{0}+t h_{B}\right)-\mathcal{J}_{0}\left(I_{R}^{0}, I_{G}^{0}, I_{B}^{0}\right)}{t} \\
&= \int_{\Omega}\left(\left|\nabla I_{R}^{0}(x)\right|^{p(x)-2} \nabla I_{R}^{0}(x), \nabla h_{R}(x)\right) d x \\
&+ \int_{\Omega}\left(\left|\nabla I_{G}^{0}(x)\right|^{p(x)-2} \nabla I_{G}^{0}(x), \nabla h_{G}(x)\right) d x \\
& \quad+\int_{\Omega}\left(\left|\nabla I_{B}^{0}(x)\right|^{p(x)-2} \nabla I_{B}^{0}(x), \nabla h_{B}(x)\right) d x
\end{aligned}
$$

Arguing in a similar manner, it can be shown that (see [2, Section A 14] for the details)

$$
\begin{aligned}
& \lim _{t \rightarrow 0} \frac{\mathcal{J}_{1}\left(I_{R}^{0}+t h_{R}, I_{G}^{0}+t h_{G}, I_{B}^{0}+t h_{B}\right)-\mathcal{J}_{1}\left(I_{R}^{0}, I_{G}^{0}, I_{B}^{0}\right)}{t} \\
& =\alpha \int_{\Omega}\left|\left(\theta^{\perp}, \nabla I_{R}^{0}\right)\right|^{\alpha-2}\left(\theta^{\perp}, \nabla I_{R}^{0}\right)\left(\theta^{\perp}, \nabla h_{R}\right) d x \\
& +\alpha \int_{\Omega}\left|\left(\theta^{\perp}, \nabla I_{G}^{0}\right)\right|^{\alpha-2}\left(\theta^{\perp}, \nabla I_{G}^{0}\right)\left(\theta^{\perp}, \nabla h_{G}\right) d x \\
& \quad+\alpha \int_{\Omega}\left|\left(\theta^{\perp}, \nabla I_{B}^{0}\right)\right|^{\alpha-2}\left(\theta^{\perp}, \nabla I_{B}^{0}\right)\left(\theta^{\perp}, \nabla h_{B}\right) d x .
\end{aligned}
$$

Setting

$$
\Lambda=\theta^{\perp}\left(\theta^{\perp}\right)^{t}=\left[\begin{array}{ccc}
\theta_{1}^{\perp} \theta_{1}^{\perp} & \theta_{1}^{\perp} \theta_{2}^{\perp} \\
\theta_{2}^{\perp} \theta_{1}^{\perp} & \theta_{2}^{\perp} \theta_{2}^{\perp}
\end{array}\right],
$$

the Gâteaux differential of $\mathcal{J}_{1}$ can be rewritten as follows

$$
\begin{aligned}
& \lim _{t \rightarrow 0} \frac{\mathcal{J}_{1}\left(I_{R}^{0}+t h_{R}, I_{G}^{0}+t h_{G}, I_{B}^{0}+t h_{B}\right)-\mathcal{J}_{1}\left(I_{R}^{0}, I_{G}^{0}, I_{B}^{0}\right)}{t}=\alpha \int_{\Omega}\left|\left(\theta^{\perp}, \nabla I_{R}^{0}\right)\right|^{\alpha-2}\left(\Lambda \nabla I_{R}^{0}, \nabla h_{R}\right) d x \\
&+\alpha \int_{\Omega}\left|\left(\theta^{\perp}, \nabla I_{G}^{0}\right)\right|^{\alpha-2}\left(\Lambda \nabla I_{G}^{0}, \nabla h_{G}\right) d x \\
& \quad+\alpha \int_{\Omega}\left|\left(\theta^{\perp}, \nabla I_{B}^{0}\right)\right|^{\alpha-2}\left(\Lambda \nabla I_{B}^{0}, \nabla h_{B}\right) d x .
\end{aligned}
$$

As for the rest terms in the cost functional $\mathcal{J}: \Xi \rightarrow \mathbb{R}$, we have the following representation for their Gâteaux derivatives (for the proof and its substantiation we refer to [13, Section 3]).

Proposition 6.1. Let $H: S_{H} \rightarrow \mathbb{R}^{3}$ and $L: S_{L} \rightarrow \mathbb{R}^{4}$ be given images such that their spectral energies satisfy conditions (4.1) Then the functionals $\mathcal{J}_{2}, \mathcal{J}_{3}$ : 
$L^{2}\left(\Omega ; \mathbb{R}^{3}\right) \rightarrow \mathbb{R}$ are convex and Gâteaux differentiable in $L^{2}\left(\Omega ; \mathbb{R}^{3}\right)$ with

$$
\begin{aligned}
\mathcal{J}_{2}^{\prime}\left(I^{0}\right)[h] & =2 \sum_{A \in\{R, G . B\}} \int_{\Omega} \alpha_{A}\left(\alpha_{R} I_{R}^{0}+\alpha_{G} I_{G}^{0}+\alpha_{B} I_{B}^{0}-Y_{H}\right) h_{A} d x, \\
\mathcal{J}_{3}^{\prime}\left(I^{0}\right)[h] & =2 \sum_{A \in\{R, G . B\}} \int_{\Omega} \Pi_{L}\left(\left[\mathcal{K} * I_{A}^{0}\right]-L_{A}\right)\left[\mathcal{K} * h_{A}\right] d x \\
& =2 \sum_{A \in\{R, G . B\}} \int_{\Omega} \Pi_{L}\left[\mathcal{K}^{*} *\left(\left[\mathcal{K} * I_{A}^{0}\right]-L_{A}\right)\right] h_{A} d x,
\end{aligned}
$$

for all $h=\left(h_{R}, h_{G}, h_{B}\right) \in L^{2}\left(\Omega ; \mathbb{R}^{3}\right)$.

We are now in a position to derive an optimality system for a unique minimizer $\left(I_{R}^{0}, I_{G}^{0}, I_{B}^{0}\right) \in \Xi \subset B V\left(\Omega ; \mathbb{R}^{3}\right)$ to constrained minimization problem (5.1). Following the standard technique which is based on the use of the Lagrange principle [10] and utilizing Proposition 4.4, we arrive at the following result.

Theorem 6.2. Let $\left(I_{R}^{0}, I_{G}^{0}, I_{B}^{0}\right) \in \Xi$ be a minimizer of constrained minimization problem (5.1). Then the following relations hold true

$$
\begin{gathered}
-\operatorname{div}\left(\left|\nabla I_{A}^{0}(x)\right|^{p(x)-2} \nabla I_{A}^{0}(x)\right)-\gamma \alpha \operatorname{div}\left(\left|\left(\theta^{\perp}, \nabla I_{A}^{0}\right)\right|^{\alpha-2} \Lambda \nabla I_{A}^{0}\right) \\
+2 \lambda \alpha_{A}\left(\alpha_{R} I_{R}^{0}+\alpha_{G} I_{G}^{0}+\alpha_{B} I_{B}^{0}-Y_{H}\right) \\
+2 \mu \Pi_{L}\left[\mathcal{K}^{*} *\left(\left[\mathcal{K} * I_{A}^{0}\right]-L_{A}\right)\right]=0 \quad \text { a.e. in } \Omega, \\
0 \leqslant I_{A}^{0} \leqslant \max _{\left(x_{i}, y_{j}\right) \in S_{L}} L_{A}\left(x_{i}, y_{j}\right) \quad \text { a.e. in } \Omega, \\
\left(\nabla I_{A}^{0}, \nu\right)=0 \quad \text { on } \partial \Omega,
\end{gathered}
$$

for $A \in\{R, G, B\}$.

Remark 6.1. In practical implementation, it is reasonable to define an optimal triplet $\left(I_{R}^{0}, I_{G}^{0}, I_{B}^{0}\right)$ using a 'gradient descent' strategy. Following the standard procedure, we can start from some initial RGB-components $\left(I_{R}^{*}, I_{G}^{*}, I_{B}^{*}\right)$ and then to solve the following initial value problem for the system of quasi-linear parabolic equations with $2 D$ elliptic operators in their principle part and Nuemann boundary conditions

$$
\begin{aligned}
& \frac{\partial I_{R}^{0}}{\partial t}-\operatorname{div}\left(\left|\nabla I_{R}^{0}(x)\right|^{p(x)-2} \nabla I_{R}^{0}(x)\right)=\gamma \alpha \operatorname{div}\left(\left|\left(\theta^{\perp}, \nabla I_{R}^{0}\right)\right|^{\alpha-2} \Lambda \nabla I_{R}^{0}\right) \\
& -2 \lambda \alpha_{R}\left(\alpha_{R} I_{R}^{0}+\alpha_{G} I_{G}^{0}+\alpha_{B} I_{B}^{0}-Y_{H}\right)-2 \mu \Pi_{L}\left[\mathcal{K}^{*} *\left(\left[\mathcal{K} * I_{R}^{0}\right]-L_{R}\right)\right], \\
& \frac{\partial I_{G}^{0}}{\partial t}-\operatorname{div}\left(\left|\nabla I_{G}^{0}(x)\right|^{p(x)-2} \nabla I_{G}^{0}(x)\right)=\gamma \alpha \operatorname{div}\left(\left|\left(\theta^{\perp}, \nabla I_{G}^{0}\right)\right|^{\alpha-2} \Lambda \nabla I_{G}^{0}\right) \\
& -2 \lambda \alpha_{G}\left(\alpha_{R} I_{R}^{0}+\alpha_{G} I_{G}^{0}+\alpha_{B} I_{B}^{0}-Y_{H}\right)-2 \mu \Pi_{L}\left[\mathcal{K}^{*} *\left(\left[\mathcal{K} * I_{G}^{0}\right]-L_{G}\right)\right], \\
& \frac{\partial I_{B}^{0}}{\partial t}-\operatorname{div}\left(\left|\nabla I_{B}^{0}(x)\right|^{p(x)-2} \nabla I_{B}^{0}(x)\right)=\gamma \alpha \operatorname{div}\left(\left|\left(\theta^{\perp}, \nabla I_{B}^{0}\right)\right|^{\alpha-2} \Lambda \nabla I_{B}^{0}\right) \\
& -2 \lambda \alpha_{B}\left(\alpha_{R} I_{R}^{0}+\alpha_{G} I_{G}^{0}+\alpha_{B} I_{B}^{0}-Y_{H}\right)-2 \mu \Pi_{L}\left[\mathcal{K}^{*} *\left(\left[\mathcal{K} * I_{B}^{0}\right]-L_{B}\right)\right],
\end{aligned}
$$




$$
\begin{gathered}
\left(\nabla I_{R}^{0}, \nu\right)=0,\left(\nabla I_{G}^{0}, \nu\right)=0,\left(\nabla I_{B}^{0}, \nu\right)=0 \quad \text { on } \partial \Omega, \\
0 \leqslant I_{A}^{0} \leqslant \max _{\left(x_{i}, y_{j}\right) \in S_{L}} L_{A}\left(x_{i}, y_{j}\right) \quad \text { a.e. in } \Omega \forall A \in\{R, G, B\}, \\
I_{R}^{0}(0, x)=I_{R}^{*}, I_{G}^{0}(0, x)=I_{G}^{*}, I_{B}^{0}(0, x)=I_{B}^{*}, \quad \forall x \in \Omega,
\end{gathered}
$$

where we propose to take the triplet $\left(I_{R}^{*}, I_{G}^{*}, I_{B}^{*}\right)$ as a result bicubic interpolation of the MODIS-like image $L: S_{L} \rightarrow \mathbb{R}^{4}$ onto the entire domain $\Omega$.

\section{Numerical Experiments}

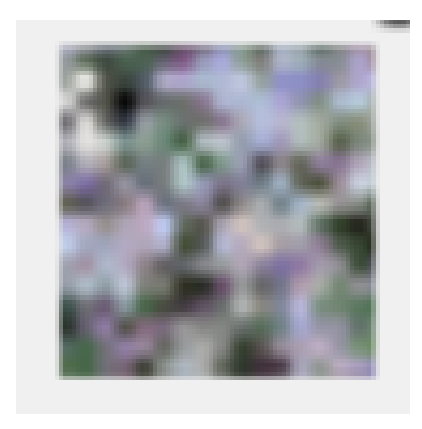

Fig. 7.1. The MODIS image with resolution 350m/pixel

In order to illustrate the proposed algorithm for the spatial increasing resolution problem of MODIS-like multi-spectral images via their fusion with Lansat-like imagery at higher resolution. As input data we have used a MODIS image of some region with resolution $350 \mathrm{~m} /$ pixel (see Fig. 7.1). This region represents a typical agricultural area with medium sides fields of various shapes.

We also have the image of the same territory with resolution $25 \mathrm{~m} /$ pixel that was made by Lansat satellite at higher resolution. Figure 7.2 shows the spectral channels of this image.

Figure 7.3 displays the reconstructed images corresponding to the data given by Figures 7.1 and 7.2. In order to validate the obtained result, we have provided the following calculations.

- Closednees of the means $\rho_{2}=|\operatorname{Mean} I-\operatorname{Mean} L|=0$;

- Closedness of the variances $\rho_{3}=100 \frac{|\operatorname{Var} I-\operatorname{Var} L|}{\operatorname{Var} L} \approx 6 \%$;

- ERGAS metric

$$
E R G A S=100 \frac{h}{l} \sqrt{\frac{1}{3} \sum_{k=1}^{3}\left(\frac{\mathrm{RMSE}(\mathrm{k})}{\mu_{0}(k)}\right)^{2}}=2.24,
$$

where $h / l$ is the ratio between the size of the high spatial resolution image pixel and the size of the pixel in the MODIS-like image. 


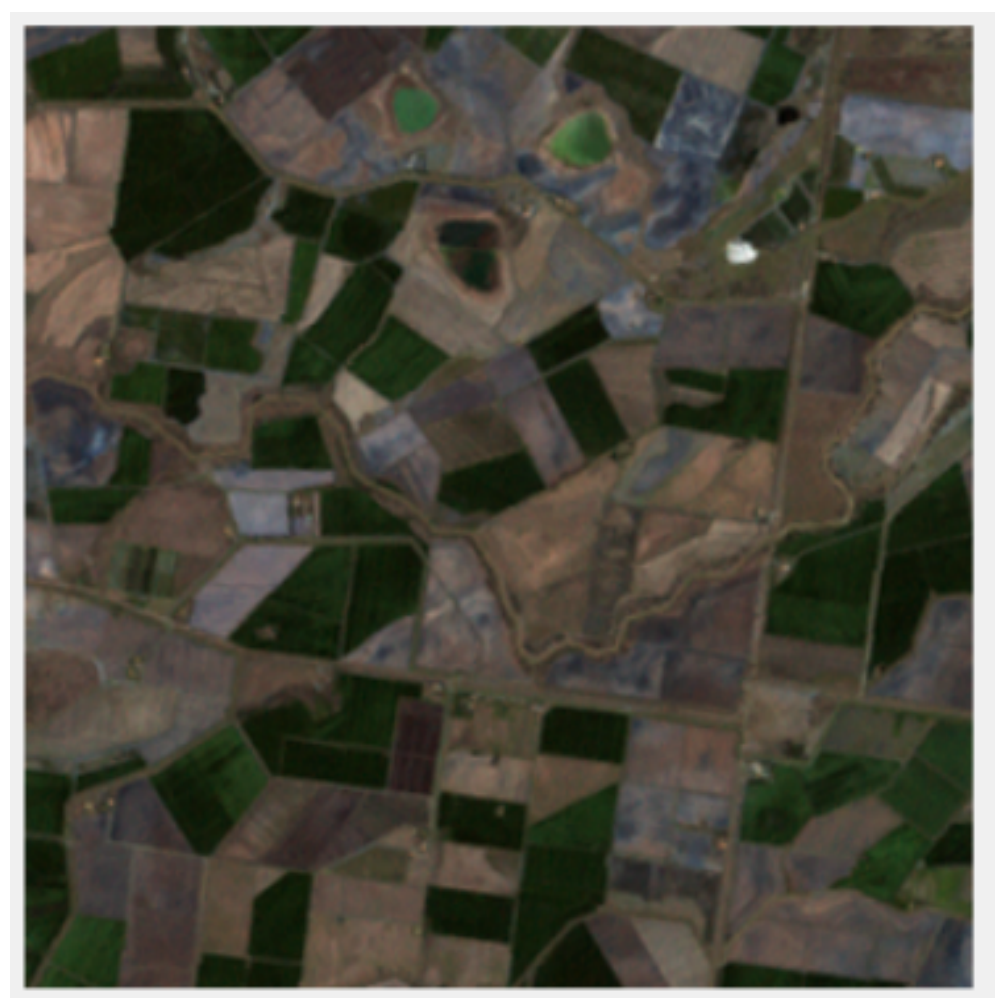

Fig. 7.2. The Lansat image with resolution $25 \mathrm{~m} /$ pixel

It is worth to notice that in view of the suggestions of Prof. L. Wald, if the ERGAS value is less than 3, the spectral quality of an image is satisfactory.

\section{References}

1. L. Ambrosio, N. Fusco, D. Pallara, Functions of bounded variation and free discontinuity problems, Oxford University Press, New York, 2000.

2. A.B. Al'shin, M.O. Korpusov, A.G. Sveshnikov, Blow-up in Nonlinear Sobolev Type Equations, De Gruyter series in nonliniear analysis and applications; 15, Walter de Gruyter GmbH \& Co, Berlin, 2011.

3. F. Andreu, C. Ballester, V. Caselles, J. M. Mazón, Minimizing Total Variation Flow, Diff. and Int. Eq., 14 (2001), 321-360.

4. H. Aтtouch, G. Buttazzo, G. Michaille, Variational Analysis in Sobolev and BV Spaces: Applications to PDEs and Optimization, SIAM, Philadelphia, 2006.

5. C. Ballester, V. Caselles, L. Igual, J. Verdera, B. Rougé, A Variational Model for P $+X S$ Image Fusion, International Journal of Computer Vision, 69 (2006), 43-58.

6. G. Crasta, V. De Cicco, Anzellotti's pairing theory and the Gauss-Green theorem, Advances in Mathematics, 343 (5) (2019), 935-970.

7. D.V. Cruz-Uribe, A. Fiorenza, Variable Lebesgue Spaces: Foundations and Harmonic Analysis, Birkhäuser, New York, 2013. 


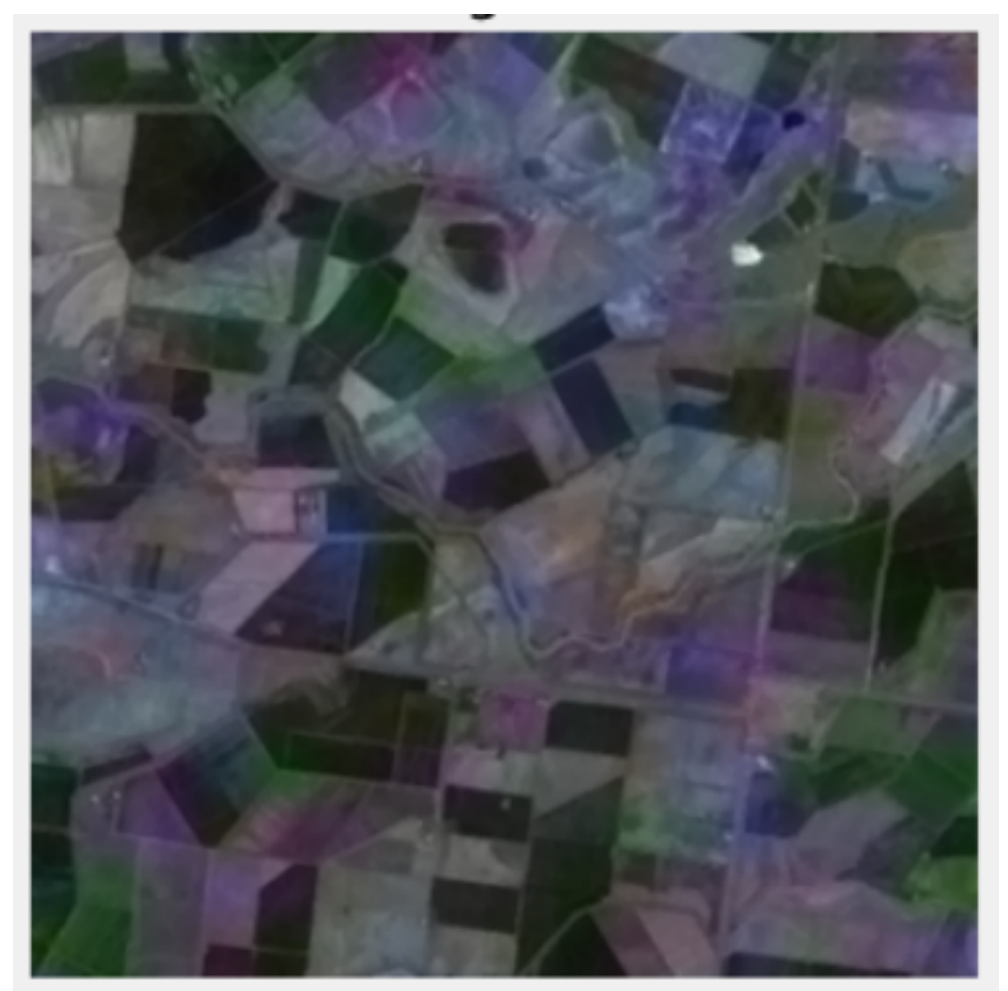

Fig. 7.3. The retrieved image at high resolution $25 \mathrm{~m} /$ pixel following the proposed approach

8. L. Diening, P. Harjulehto, P. HÄstö, M. RüẐIĈKA, Lebesgue and Sobolev Spaces with Variable Exponents, Springer, New York, 2011.

9. D. Frantz, Landsat+ Sentinel-2 analysis ready data and beyond, Remote Sens., 11, 1124, 2019.

10. A.D. Ioffe, V.M. Tikhomirov, Theory of Extremal Problems, North Holland, Amsterdam, 1979.

11. V.V. Hnatushenko, P.I. Kogut, M.V. Uvarov, On Flexible Co-Registration of Optical and SAR Satellite Images, in "Lecture Notes in "Computational Intelligence and Decision Making" (series "Advances in Intelligent Systems and Computing Editors: Babichev, S., Lytvynenko, V., Wrijcik, W., Vyshemyrskaya, S. (Eds.) ), Springer, 2021, 515-534.

12. V.V. Hnatushenko, P.I. Kogut, M.V. Uvarov, Variational Approach for Rigid Co-Registration of Optical/SAR Satellite Images in Agricultural Areas, Journal of Computational and Applied Mathematics, to appear.

13. V.V. Hnatushenko, P.I. Kogut, M.V. Uvarow, On optimal 2-D domain segmentation problem via piecewise smooth approximation of a selective target mapping, J. of Optimization, Differential Equations and Their Applications (JODEA), 27 (2) (2019), 39-74.

14. B. Kawohl, F. SchuRICHT, Dirichlet problems for the 1-Laplace operator, including the eigenvalue problem, Communications in Contemporary Mathematics, 9 (4) (2007), 515-543. 
15. P.I. Kogut, G. Leugering, Optimal Control Problems for Partial Differential Equations on Reticulated Domains. Approximation and Asymptotic Analysis, Series: Systems and Control, Birkhäuser Verlag, Boston, 2011.

16. D.P. RoY, J. Li, H.K. Zhang, L. YAn, Best practices for the reprojection and resampling of Sentinel-2 Multi Spectral Instrument Level $1 C$ data, Remote Sens. Lett., 7 (2016), 1023-1032.

17. D.P. Roy, H. Huang, L. Boschetti, L. Giglio, H.K. Zhang, J. Li, Landsat8 and Sentinel-2 burned area mapping - a combined sensor multi-temporal change detection approach, Remote Sens. Environ., 231, 111254, 2019.

18. L.I. Rudin, S. Osher, E. FATEmi, Nonlinear total variation based noise removal algorithms, Physica, 60(D) (1992), 259-268.

19. L. Yan, D.P. Roy, H. Zhang, J. Li, H. Huang, An automated approach for sub-pixel registration of Landsat-8 Operational Land Imager (OLI) and Sentinel-2 Multi Spectral Instrument (MSI) imagery, Remote Sens., 8, 520, 2016.

20. V.V. ZHikov, Solvability of the three-dimensional thermistor problem, Proceedings of the Steklov Institute of Mathematics, 281 (2008), 98-111.

21. V.V. ZHIKOV, On variational problems and nonlinear elliptic equations with nonstandard growth conditions, Journal of Mathematical Sciences, 173(5) (2011), $463-570$. 